espacio abierto 



\title{
La práctica pedagógica en la investigación en educación matemática desde la perspectiva de los egresados ${ }^{1}$
}

\author{
Sonia Valbuena Duarte ${ }^{2}$ \\ Universidad del Atlántico, Colombia \\ soniabalbuena@mail.uniatlantico.edu.co \\ Robinson Junior Conde Carmona ${ }^{3}$ \\ Universidad del Atlántico, Colombia \\ rjconde@mail.uniatlantico.edu.co \\ Iván Andrés Padilla Escorcia ${ }^{4}$ \\ Universidad del Atlántico, Colombia \\ iapadilla@mail.uniatlantico.edu.co \\ Recibido: 21 de octubre de 2017 \\ Aceptado: 18 de junio de 2018 \\ Disponible en línea: 28 de diciembre de 2018
}

1 Este artículo se desprende de la investigación titulada "Caracterización de la actividad de investigación en educación matemática y su relación con las prácticas pedagógicas en la licenciatura en Matemáticas”, realizada entre junio del 2016 y junio del 2017, en la Universidad del Atlántico.

2 Doctorando en Educación. Magister en Educación y Magister en Matemáticas. Docente de tiempo completo y coordinadora del programa de Licenciatura en Matemáticas, Universidad del Atlántico.

3 Doctorando en Educación Matemática, especialista en Métodos Numéricos Aplicados, licenciado en Matemáticas, Universidad del Atlántico. Docente de la Universidad de la Costa -CUC-y de American School.

4 Estudiante de la especialización Estadística Aplicada, licenciado en Matemáticas, Universidad del Atlántico. Docente de tiempo completo del Colegio Americano de Barranquilla. 


\title{
La práctica pedagógica en la investigación en educación matemática desde la perspectiva de los egresados
}

\section{Resumen}

Este texto tuvo como objetivo describir la práctica pedagógica del formador de formadores como actuación comunicativa en su relación con la investigación en educación matemática en un programa que forma docentes de matemáticas. Para recabar la información se hizo uso de diversas técnicas e instrumentos; dentro de estas, anotamos aquí: observaciones no participantes, registros en diarios de campo y entrevista a los docentes a cargo de las asignaturas referentes a investigación del programa académico y a docentes ya egresados del mismo programa que ejercen la profesión en una institución educativa. Esto, para analizar, desde diferentes puntos de vista, la progresión y el alcance en que se encuentra el programa en la formación en investigación desde la práctica pedagógica, y compararlo con los referentes que la literatura reporta como parte fundante en la formación y requerimientos de un docente investigador, quien se forma a través de la práctica pedagógica que establece la propuesta curricular de una licenciatura en Matemáticas en Colombia.

Palabras clave: práctica pedagógica; práctica investigativa; maestro en formación inicial; docente de matemáticas; investigador; investigación en educación matemática

\section{Pedagogical practice in the research in mathematics education from the perspective of the alumni}

\begin{abstract}
The purpose of this research project was to describe the pedagogical practice of teacher trainers as a communicative action in relation to the research in mathematics education in a math teacher training program. In order to gather the information, various techniques and instruments were used, including non-participant observations, records in fieldwork diaries and interviews with teachers in charge of subjects related to research in the academic program, as well as with teachers who have finished the program and that are currently working in an educational institution. These actions were carried out in order to analyze, from different points of view, the progression and scope of the program in research training from the pedagogical practice, and compare it with the references found in literature where research training is a founding part in the training and requirements of a research teacher, who is formed through the pedagogical practice established by a curricular proposal of an undergraduate program in Mathematics in Colombia. Keywords: pedagogical practice; research practice; teacher initial education; mathematics teacher; researcher; research in mathematics education
\end{abstract}

\section{A prática pedagógica na pesquisa em ensino matemático desde a perspectiva dos graduados}

\section{Resumo}

O objetivo desta pesquisa foi descrever a prática pedagógica do formador de formadores como ação comunicativa em relação à pesquisa em Educação Matemática em um programa de formação de professores de Matemática. Para a coleta de informações foram utilizadas várias técnicas e instrumentos. Dento delas anotamos: observações não participantes, registros em diários de campo e entrevistas com docentes responsáveis por matèrias relacionadas à pesquisa no Programa Acadêmico, a professores já graduados do programa e que praticam a profissão em uma instituição de ensino. Isso foi para analisar desde diferentes pontos de vista a progressão e o escopo em que o Programa está no que diz respeito a formação em pesquisa a partir da prática pedagógica, e compará-la com as referências de literatura reportadas em formação e requerimentos de um docente que é formado por meio da prática pedagógica que estabelece a proposta curricular de uma licenciatura em prática matemática.

Palavras-chave: prática pedagógica; prática investigativa; docente em formação inicial; docente de matemática; pesquisador; pesquisa em educação matemática 


\section{Introducción}

Existe un interés en América Latina y en el mundo por mejorar la formación docente, la formación inicial, continua, y por involucrar al docente en las politicas que promuevan el fortalecimiento de la profesión (Vaillant, 2016).

La docencia es una de las profesiones más valiosas y gratificantes que existen; formar diferentes profesionales, tales como ingenieros, arquitectos, abogados, médicos, entre otros, es de vital importancia para el desarrollo de un país. Por otra parte, el docente es visto como agente de transformación social y líder gestor de cambio, que, al dominar su disciplina y a través de metodologias activas, ofrece herramientas necesarias para que los estudiantes comprendan el mundo desde diversos lenguajes, aprendan a vivir con los demás y sean productivos (Garcés, 2010). Para el Ministerio de Educación Nacional (MEN) (2005), el trabajo del docente suele entrañar, en algunas ocasiones, un vaivén en el que son muchos los factores que conducen al éxito o fracaso de los centros educativos. Por ello, se plantea que un elemento que asegura la constante renovación de un docente es el fortalecimiento en su práctica investigativa, como una actividad inherente al proceso educativo mismo, ya sea a través de investigación científica o formativa (Restrepo, 2005).

En ese orden de ideas, la investigación permite interpretar el mundo educativo, generar reflexiones en el profesor y aportar elementos a su formación, lo cual constituye un proceso esencial si se busca alcanzar desarrollos en la educación, en la transformación de las prácticas, en el cambio social y, particularmente, en la formación del profesor (Sánchez-Robayo y Torres-Duarte, 2017). Por tal motivo, la investigación es un componente que enriquece la práctica pedagógica del educador y que potencia la cualificación docente, incrementando la productividad en su vida académica (Acuña, 2015).

La investigación desde su propia praxis impacta el quehacer del docente; impacta su metodologia, sus estrategias didácticas. 


\begin{abstract}
En definitiva, da lugar a clases motivantes y transformadoras para el estudiante. En esta misma línea, Godino (2011) expresa que las teorias expuestas permiten ver la importancia que, a lo largo de los años, ha ido ganando la investigación como herramienta para fortalecer la práctica pedagógica del docente a partir de las experiencias del aula. Para ello, resulta fundamental que la relación entre las prácticas pedagógicas e investigativas se haga protagonista del proceso de formación y evidenciable en las escuelas que buscan promover una educación exitosa y de calidad.
\end{abstract}

Pese a lo planteado, son pocos los docentes que asumen roles investigativos, pues muchos de ellos se conforman con cumplir los requisitos que les posibilitan optar por un título profesional como licenciado en cualquier área del conocimiento y se apartan del desarrollo de proyectos investigativos que les permitirian ampliar su currículo. Asumen, de este modo, una posición cómoda que los aleja de la esencia de la práctica docente, que es un proceso de aprendizaje diario y, sobre todo, de atender dificultades en las que se encuentran las escuelas en muchas ocasiones, lo cual podría adelantarse a partir de un pensamiento crítico que permita conectar la investigación con la práctica, a fin de introducir cambios comprometidos con la mejora del proceso de formación inicial de maestros (Valbuena, Conde y Ortíz, 2018a).

Ahora bien, cabe resaltar que el docente que investiga es una persona que decide transformar su realidad, problematizando y abordando aquellas situaciones que le han generado dudas y sobre las cuales actúa (Sánchez-Robayo y Torres-Duarte, 2017). Esto, en referencia a los procesos educativos, lo hace partir de reflexiones y experiencias que se dan en el salón de clases, teniendo en cuenta que el profesor es quien impacta de manera significativa el mundo de la escuela (Stenhouse citado en Saker García, 2014), y que la educación debe contribuir al desarrollo integral del ser humano para que cada individuo comprenda las demandas de su contexto, favorezca apuestas de desarrollo social y, en definitiva, contribuya a forjar una mejor sociedad. 
Bajo esa perspectiva, el presente texto tiene el interés de describir la relación que existe entre la investigación y la práctica pedagógica, desde el discurso que manejan los egresados de un programa de formación de licenciados en Matemáticas, en la Universidad del Atlántico, Colombia, y la experiencia investigativa de los mismos. Ello, atendiendo al hecho de que una de las tareas relevantes que debe asumir la educación matemática es el desarrollo tanto de maestros en formación inicial como de maestros en ejercicio, y asumiendo que la investigación en educación matemática es un campo de investigación transdisciplinaria (Arboleda y Castrillón, 2007).

Es clara la necesidad de que los docentes de formadores manejen un discurso y una práctica pedagógica a partir de la solución de problemas y proyectos investigativos que impacten y motiven en el contexto del educando (Hernández, 2009). El proceso de investigar-enseñar-aprender es inherente, debido a diversas razones que pueden ir desde la especificidad contractual entre la universidad y el docente, hasta el interés y perfil del mismo, entre otras posibilidades. Por tal motivo, se encuentra con gran frecuencia que algunos maestros dedican sus tiempos de trabajo solo a investigar y otros solo a dictar una clase. Esto, basados en sus intereses particulares (Acuña, 2015).

La necesidad expresada radica en tener en cuenta que los docentes de un programa de licenciatura y, particularmente, de uno en Matemáticas -que es el interés del trabajo investigativo que dio lugar a este texto- son formadores de personas que en un futuro también lo serán. Es decir, son formadores de formadores. Ante esto se requiere que los docentes estén armados de una preparación sobresaliente que les sirva de base para desenvolverse en la práctica docente, ya que es necesario relacionar los modelos teóricos de formación del profesorado con su práctica profesional (Badillo, Font y Martínez, 2013). En el ámbito educativo existen docentes en formación inicial, en ejercicio reciente, de varias décadas de desempeño laboral, replicando las mismas prácticas con las cuales fueron formados. Esto nos lleva a presuponer la 
gran influencia que representa para cada docente la forma como se presenta la tarea educativa, lo cual muestra, a la larga, un estancamiento en la práctica docente (Valbuena Duarte, Conde Carmona y Ortíz Ortíz, 2018b).

Así mismo, y de acuerdo con Llinares (2014), damos cuenta de algunas habilidades con las que debe contar un docente:

- La capacidad de relacionar las tareas y actividades que diseñan y los objetivos alcanzados durante la intervención en el aula.

- La capacidad para identificar y analizar evidencias de aprendizaje matemático de los niños en aras de evaluar y revisar la intervención diseñada y hacerla más efectiva.

De ese modo, este texto se justifica, entonces, a partir de la realidad que atiende, que no es otra que la búsqueda de pautas que contribuyan con una formación de calidad para los docentes de matemáticas, con el fin de que investiguen su propia praxis y de que los resultados de sus pesquisas redunden en la calidad de su misma práctica pedagógica. Todo ello, en definitiva, en aras de desarrollar la capacidad de sus estudiantes para aprender los saberes específicos del área de matemáticas, ya que la investigación educativa no puede definirse solamente como resolver problemas teóricos, sino que debe operar dentro del marco de los fines prácticos conducidos al fortalecimiento de actividades educativas (Carr y Kemmis citados en Saker García, 2014).

\section{Teorias que sustentan la investigación}

E1 Ministerio de Educación Nacional en Colombia sostiene, en los Lineamientos de calidad para las licenciaturas en educación (2015), que el campo propio de la investigación debe ser transversal y se define por los desarrollos teóricos y empíricos relacionados con la educación, la formación de maestros y la interacción 
entre pedagogia, didáctica, disciplina e investigación para producir nuevas formas de pensar las prácticas. El MEN (2014) señala que la práctica pedagógica debe ser concebida como eje central de formación, según los Lineamientos de calidad, lo que se constituyó en una razón de orden práctico para el proyecto del que se desprende este artículo y justificó priorizar la investigación sobre las prácticas pedagógicas en pro de buscar un mejoramiento en la calidad de las mismas.

Esto, dado que los docentes, como profesionales expertos, trabajan colaborativamente en la construcción del conocimiento de su área, por medio de la concepción de propuestas pedagógicas que sirven como espacios de reflexión, cuestionamiento y observación para fomentar la innovación educativa. Se denota así la relevancia de la investigación como medio que genera impacto en la práctica de los docentes, y en particular de los de matemáticas, pues esta fortalece sus competencias científicas (TejadaFernández, Carvalho-Dias y Ruiz-Bueno, 2017).

Ahora bien, bajo la normatividad nacional, el MEN (2016) promulgó la Resolución N. 02041 el 3 de febrero del 2016, la cual aplica a los programas académicos de licenciaturas concordantes con la fecha de radicación de solicitud para acreditación de alta calidad del programa. Esta resolución tuvo modificaciones y fue derogada por la resolución 19583 del 15 de septiembre de 2017, pero en todo caso continúa contemplando los lineamientos de calidad para las licenciaturas. De esta se resalta la búsqueda de la mejoría significativa en las competencias docentes en todo su quehacer pedagógico y profesional, dado que promueve que los maestros sean partícipes de actividades de investigación formativa y en el aula. Además, se espera que los educadores de las licenciaturas hagan investigación disciplinar y pedagógica, y que aporten a la producción de conocimiento relevante, con el fin de que hagan parte de la comunidad académica internacional en su área y de que estén en capacidad de orientar los procesos de formación de los futuros licenciados. Esto hace alusión a que es necesaria una buena preparación por parte del grupo de docentes 
que asuma el rol de asignaturas de investigación en el programa, de suerte que sean un ejemplo de motivación, esfuerzo y alto nivel académico en lo referente a la construcción de investigación desde el aula y, a su vez, esto se vea proyectado por medio de las investigaciones que elaboren -artículos científicos, libros, ponencias, entre otras-.

Por otro lado, el Decreto 2450 del 17 de diciembre de 2015 "Por el cual se reglamentan las condiciones de calidad para el otorgamiento y la acreditación de los Programas académicos de licenciatura y los enfocados a la educación" del MEN (2015), exige que en los programas haya un núcleo de profesores con experiencia acreditada en investigación, orientada de manera especial a mejorar los procesos de enseñanza y aprendizaje, con formación de maestría o doctorado, y cualidades y tiempo de dedicación destinado al acompañamiento del estudiante en actividades académicas de investigación y en prácticas pedagógicas y educativas. Sin embargo, lo que se evidencia de manera casi que general, cuando se hace un barrido de los docentes mirando sus CVLAC, es que esto no está sucediendo en el programa, ya que no existen muchos registros de docentes que cumplan con lo anterior, y son muy escasos los que cuentan con un proceso investigativo calificado que los haga resaltar.

Por lo tanto, abrimos un par de incógnitas apoyándonos en Acuña (2015): ¿Qué condiciones posibilitan la investigación de los educadores? ¿Es posible enseñar investigación sin ser investigador? Estos cuestionamientos nos dan a entender que la puesta en escena que vienen presentando los docentes de investigación de la licenciatura en Matemáticas no es la mejor, lo cual se refleja en el poco dominio investigativo con el que cuentan los maestros en formación inicial y los recientes egresados del programa.

De este modo, el MEN (2014), en el documento Sistema colombiano de educación de formadores y lineamientos de politica, establece que mejorar la calidad de la educación está directamente relacionado con la calidad de los docentes y, de manera particu- 
lar, con su formación inicial. En lo referente a la formación inicial de los maestros, se expresa que esta es un componente necesario para que predomine la calidad en un sistema educativo, con lo cual resulta relevante este texto, porque, por un lado, representa una búsqueda de alternativas que le permitan al docente de matemáticas del programa reflexionar acerca de su proceso investigativo, $\mathrm{y}$, por otro, porque sirve como estrategia para aportar al mejoramiento de las prácticas educativas, con el fin de contribuir con la calidad de la educación en todos los niveles de actuación del licenciado en matemáticas. En este artículo se consideran las prácticas pedagógicas en educación utilizadas por los educadores, desde su discurso, y fundamentadas en una perspectiva investigativa que le ayude tanto al futuro docente de matemáticas como al formador de formadores a consolidar la investigación como un elemento importante en la docencia.

Por otro lado, teniendo en cuenta que la formación es un proceso individual en el cual el profesor modifica y desarrolla sus estructuras a partir de sus propias experiencias, se hace evidente la necesidad de que la relación entre los docentes formadores y los maestros en formación inicial se haga real desde las experiencias que los docentes viven, las cuales, de una u otra manera, contribuyen a una formación de calidad (Riscanevo Espitia y Jiménez Espinosa, 2017).

Cabe resaltar, además, que la licenciatura en Matemáticas se encuentra en un proceso de acreditación, por lo que se vuelve de suma importancia contar con un personal docente cualificado. En las directrices del MEN (2016) propuestas en la Resolución 02041 del 2016, este es uno de los argumentos esenciales. En ese sentido, la proyección de este artículo aportará a dilucidar la importancia y los roles que debe tener un docente del siglo XXI. Lo que quiere decir que un docente de la actualidad debe ser proactivo y capaz de encontrar alternativas que le permitan asumir el papel de líder de la clase, en búsqueda de brindarles a sus estudiantes las mejores condiciones posibles de eficacia durante la misma. Así, Edo y Badillo (2014) resaltan que se busca que los futuros 
maestros desarrollen las competencias profesionales que les permitan identificar y analizar la complejidad asociada a las situaciones matemáticas de enseñanza-aprendizaje.

\section{Teorias alrededor de la educación matemática}

En el programa de licenciatura en Matemáticas son muchos los factores que influyen en la deserción de los estudiantes que ingresan a hacer parte del alma mater; algunos deciden abandonar por completo la universidad y otros solo se cambian de programa. Una cantidad considerable de estudiantes ingresa al programa académico como una segunda opción que el sistema de admisión a la universidad les permite inscribir, lo que relativiza la importancia que pueda tener esta carrera profesional para ellos.

Lo que se espera, a partir del modelo de formación por competencias, en lo referente a saber hacer, es que el conocimiento que el docente vivió en las aulas universitarias le sea útil en su vida profesional. Las competencias académicas son el reflejo de las competencias profesionales de la persona que ejerce la disciplina para la que lo prepararon los estudios universitarios (o bien están inspiradas en ellas); en este caso, la formación docente en el área del conocimiento de las matemáticas (Pochulu, Font y Rodríguez, 2016). Por tal motivo, algunos autores como Schoenfeld y Kilpatrick (2008) aseguran que una de las principales competencias con la que debe contar un docente de matemáticas es la reflexión acerca de su propia práctica (pensar cómo se desarrolló una situación en clase y qué se podría hacer para mejorarla). Por otra parte, Fernández, Llinares y Valls (2012) consideran que una de las competencias clave que debe tener un docente de matemáticas es la de "mirar con sentido", porque esta le permite aproximarse a las situaciones de enseñanza y aprendizaje de las matemáticas de un modo profesional y diferenciarse de la manera de ver de alguien que no es experto en el tema. Dicha competencia también está relacionada con la reflexión sobre la 
práctica que deben desarrollar los docentes para realizar procesos de instrucción de calidad. En ese orden de ideas, lo anterior conlleva a que los docentes de matemáticas de la actualidad enriquezcan su práctica a través de una reflexión propia sobre su quehacer diario.

Futuros licenciados en formación requieren de un estado emocional alto que les permita esforzarse para ser excelentes profesionales, y qué mejor ejemplo que las experiencias de sus formadores y hacedores de la práctica. Por ello, el docente formador de formadores debe ser una persona con alto compromiso profesional, con una formación y cualificación permanentes; un profesional que investigue su propia práctica y cuyos hallazgos impacten su misma praxis. Esto es, "que el ejemplo enseñe".

Sin embargo, una búsqueda en los CVLAC (plataforma utilizada por Colciencias para el registro de datos de todo profesional, como títulos, artículos científicos y formativos, libros, entre otros) de los docentes del programa, arrojó que algunos ni siquiera están registrados, lo que denota un espíritu investigativo pobre. Esto va en contravía de lo que afirman Giménez, Vanegas, Font y Rubio (2016) en lo concerniente al reto que se les presenta a los educadores matemáticos, que no es otro que reconocer cómo la enseñanza de las matemáticas está contribuyendo al logro de las metas que encierra la educación. Ello, gracias a los docentes que asumen competencias profesionales reflexivas sobre su práctica, dado que estas les permiten reconocer los cambios de la sociedad en la que se insertan. Esto solo es posible a partir del sentido de indagación o exploración con que cuenten los docentes con respecto a la educación matemática, pues son ellos quienes conocen bien la realidad de sus aulas y de sus estudiantes, y, por ello, los únicos que pueden calibrar adecuadamente los estímulos y desafios que deben darles y plantearles a sus alumnos; en el caso de la formación de docentes de matemáticas es la necesaria conexión entre crear problemas y su relación con el proceso de resolución de los mismos (Mallart Solaz, Font Moll y Malaspina, 2016). 
Por último, es de relevancia en las teorias en educación matemática lo aportado por Pocholu y Rodríguez (2012), que definen el concepto del horizonte matemático como el conocimiento que aporta a los trabajos de los docentes de matemáticas desde diferentes perspectivas. Este conocimiento es superior al conocimiento específico, que los lleva a cuestionarse lo siguiente: ¿puede tener consecuencias matemáticas conflictivas algo que se ha dicho de manera explícita o implícita? ¿Es esto interesante e importante desde el punto de vista matemático? ¿Hay alguna desviación en las ideas matemáticas tratadas? Tales interrogantes son fundamentales en la reflexión e investigación de las prácticas pedagógicas del docente de matemáticas.

\section{Metodologia}

Bajo un enfoque cualitativo-hermenéutico, el diseño metodológico utilizado en la investigación de la que resultó este artículo fue de tipo descriptivo, el que, según Hernández (2003), se refiere a la estrategia preseleccionada para poder llegar a la información que se necesita del modo más específico posible. El estudio fue de tipo exploratorio, es decir que se considera la observación de las categorías cualitativas (Hernández, 2010).

La metodología implementada en el análisis de los datos siguió la idea de Badillo, Azcárate y Font (2011) de orientarla por fases para alcanzar los objetivos antes planteados y responder las preguntas de la investigación.

De otro lado, las técnicas e instrumentos recolectados en la investigación fueron los siguientes: entrevistas a egresados, grupos focales con egresados, registros en diario de campo y bitácora de observación. 


\section{Fases para la recolección de la información}

Primera fase: elaboración del constructo "interrelación entre el discurso del docente en investigación en educación matemática y práctica pedagógica", a partir de aportes de la historia y epistemología de estos conceptos y de resultados de investigaciones previas en los desarrollos teóricos que la comunidad académica ha aportado en esta materia.

Segunda fase: 1) Transcripción de las entrevistas sobre las argumentaciones de los actores (egresados del programa de licenciatura en Matemáticas), incorporando las respuestas de cada uno a las preguntas de las mismas. 2) Triangulación de la información y convergencia para llevar a cabo el procedimiento de categorización y el análisis de la caracterización de la investigación en educación matemática y su interrelación con la práctica pedagógica, utilizando la técnica para triangular al observador, el diario de campo y las entrevistas.

Tercera fase: determinación de las características del egresado y de los desarrollos que estas tienen en el contexto de la licenciatura en Matemáticas de la Universidad del Atlántico, con el fin de diseñar estrategias que permitan la integración e interrelación entre la investigación en educación matemática y la práctica pedagógica, para aplicarlas en la licenciatura en Matemáticas en la Universidad del Atlántico.

Muestra: 10 egresados de la licenciatura en Matemáticas vinculados laboralmente a una institución educativa de la ciudad de Barranquilla.

Instrumentos de recolección: durante un periodo académico se realizaron observaciones en el espacio académico de las asignaturas de Investigación formativa y de Prácticas en la licenciatura en Matemáticas de la Universidad del Atlántico. Se hicieron, específicamente, bitácoras de observación, diarios de campo y entrevistas semiestructuradas con respuestas abiertas de docentes egresados de la licenciatura en Matemáticas que ejercen su rol en varias instituciones educativas. En cada caso se elaboró un registro y se construyó conocimiento en profundidad sobre las experiencias encontradas. 


\begin{tabular}{|c|c|c|}
\hline Categoria & $\begin{array}{c}\text { Bitácora de observación y } \\
\text { diario de campo }\end{array}$ & Entrevista \\
\hline $\begin{array}{l}\text { Investigación } \\
\text { en educación } \\
\text { matemática }\end{array}$ & $\begin{array}{l}\text { Durante las distintas observaciones } \\
\text { y diarios de campo que se aplicaron } \\
\text { al grupo de docentes egresados del } \\
\text { programa, cabe resaltar algunas } \\
\text { situaciones puntuales, como por } \\
\text { ejemplo, la de un docente que dicta } \\
\text { matemáticas en el grado sexto, que } \\
\text { promueve la indagación, exploración } \\
\text { e investigación de las temáticas an- } \\
\text { tes de explicarlas. Esto, a través de } \\
\text { lecturas y situaciones de contexto } \\
\text { en las que se aplican las temáticas. } \\
\text { En los demás docentes observados, } \\
\text { en especial en los de álgebra (octavo } \\
\text { grado de secundaria), se evidencia } \\
\text { un discurso tradicional al momento } \\
\text { de explicar las temáticas, siendo el } \\
\text { tableroy el marcador los principales } \\
\text { protagonistas (conductismo). }\end{array}$ & $\begin{array}{l}\text { De las entrevistas cabe destacar que } \\
\text { solo dos de los docentes consideran } \\
\text { que intentan asumir el rol de do- } \\
\text { centes investigadores durante sus } \\
\text { clases, muy a pesar de que piensan } \\
\text { que no recibieron la mejor formación } \\
\text { investigativa en la universidad. No } \\
\text { obstante, son conscientes del rol } \\
\text { que deben asumir al ser docentes del } \\
\text { siglo XXI, por lo cual fomentan y leen } \\
\text { acerca de estrategias que permitan } \\
\text { mejorar el ambiente del aula y las ha- } \\
\text { bilidades de sus estudiantes a través } \\
\text { de la investigación. Por otro lado, el } \\
\text { resto de docentes asumen el rol de } \\
\text { no estar preparados para introducir } \\
\text { la investigación a sus clases debido } \\
\text { a que solo le encontraron sentido a } \\
\text { esta a través de la realización de su } \\
\text { trabajo de grado de la licenciatura. } \\
\text { Sin embargo, les gustaria mejorar en } \\
\text { ese aspecto. }\end{array}$ \\
\hline $\begin{array}{c}\text { Práctica } \\
\text { pedagógica }\end{array}$ & $\begin{array}{l}\text { En esta categoria cabe destacar } \\
\text { que, a través de las observaciones } \\
\text { y del seguimiento realizados a los } \\
\text { docentes, en especial a los que } \\
\text { dictan las cátedras de matemáticas } \\
\text { en grados avanzados, como octavo, } \\
\text { noveno y décimo de secundaria, } \\
\text { estos asumian el rol de ser docentes } \\
\text { que se dedican a dar sus clases, po- } \\
\text { ner ejemplos, muchos ejercicios y, } \\
\text { por tal motivo, omiten fortalecer la } \\
\text { habilidad de análisis, razonamiento } \\
\text { e interpretación de sus estudiantes, } \\
\text { a través de problemas del diario } \\
\text { vivir en donde deban explorar e } \\
\text { indagar más allá de un algoritmo } \\
\text { matemático. }\end{array}$ & $\begin{array}{l}\text { En esta categoria los docentes afir- } \\
\text { maron que son docentes tradicio- } \\
\text { nales, en general, pues están cons- } \\
\text { cientes de que sus clases son poco } \\
\text { motivadoras y solo se prioriza en la } \\
\text { enseñanza de tablero y marcador, } \\
\text { dando poca libertad a que los estu- } \\
\text { diantes fortalezcan competencias, } \\
\text { habilidades y destrezas. No obstante, } \\
\text { la búsqueda del crecimiento de su } \\
\text { práctica docente es una invitación } \\
\text { a cambiar el paradigma de que las } \\
\text { clases de matemáticas no son es- } \\
\text { timulantes, como en el caso de dos } \\
\text { de los docentes, quienes coinciden } \\
\text { en que intentan encontrarle sentido } \\
\text { cotidiano a lo que enseñan. }\end{array}$ \\
\hline $\begin{array}{c}\begin{array}{c}\text { Relación } \\
\text { entre la }\end{array} \\
\text { investigación } \\
\text { en educación } \\
\text { matemática } \\
\text { y práctica } \\
\text { pedagógica }\end{array}$ & $\begin{array}{l}\text { A partir de las observaciones y del } \\
\text { seguimiento hecho a los docentes, se } \\
\text { pudo determinar que la mayoria no } \\
\text { reflexiona acera de su práctica y, por } \\
\text { lo tanto, no la conecta a través de un } \\
\text { rol investigativo que permita el cre- } \\
\text { cimiento de su labor docente. Ello, } \\
\text { a excepción de dos docentes que, a } \\
\text { través del fomento de la lectura y } \\
\text { de actividades dinámicas, exploran } \\
\text { las temáticas de matemáticas en el } \\
\text { contexto. }\end{array}$ & $\begin{array}{l}\text { Los docentes afirmaron en la entre- } \\
\text { vista que, teniendo en cuenta las } \\
\text { dificultades que desde la universidad } \\
\text { presentan con respecto a investigar, } \\
\text { se les hace aún más complejo relacio- } \\
\text { nar la investigación con su práctica } \\
\text { diaria, que es el ideal teórico que } \\
\text { exigen las políticas de educación en } \\
\text { Colombia; un ideal del que tienen } \\
\text { consciencia de estar lejos aún, ya que } \\
\text { en el pregrado no encontraron ense- } \\
\text { ñanzas en las que pudieran aplicar } \\
\text { de manera práctica lo teórico, y, por } \\
\text { tal razón, una constante en ellos es } \\
\text { ser docentes tradicionales. }\end{array}$ \\
\hline
\end{tabular}

Tabla 1. Hallazgos presentados por categorías de los instrumentos a los egresados del programa de licenciatura en Matemáticas

Fuente: elaboración propia. 


\section{Análisis de las observaciones, diarios de campo y entrevistas a los egresados del programa de licenciatura en Matemáticas que ejercen su profesión en una institución educativa}

A través de secciones de bitácoras de observación y diarios de campo se evidenciaron algunas experiencias de aula de diez egresados recientes del programa de licenciatura en Matemáticas que trabajan en distintas instituciones educativas de Barranquilla en los grados de secundaria. Al momento de observarlos, se tuvieron en cuenta algunos criterios, para luego analizarlos desde su práctica, esto es, a través de la relación que establecen entre su quehacer con la investigación, aparte de la promoción que le hacen a la misma a través de la enseñanza del conocimiento matemático. Lo anterior, tomado como momentos de reflexión de los docentes para responder a una entrevista relacionada con sus vivencias como estudiantes del programa, su práctica pedagógica e investigativa y la relación entre estas.

\section{Categoria: investigación en educación matemática}

Bitácora de observación: durante las observaciones realizadas a los docentes egresados del programa, se pudo apreciar que, en general, no asumen el rol de trabajar los tópicos de matemáticas a través de la exploración, la elaboración de preguntas o el abordaje de los mismos desde problemas del diario vivir. No obstante, cabe resaltar que uno de los docentes observados sí cumple con lo anterior, pues prioriza que sus estudiantes, a partir de conceptos, creen su propia definición de los temas, a diferencia de los otros docentes que asumen la enseñanza de las matemáticas de manera tradicional.

Según lo observado, se denota que los docentes egresados no ponen en práctica nociones investigativas, y desde lo propuesto por Acuña (2015), quien sostiene que la investigación es un com- 
ponente que enriquece la práctica pedagógica del educador, se evidencian los bajos estímulos con los que los estudiantes reciben las clases de matemáticas.

Diario de campo: a través de los diarios de campo se constató que una forma de promover la investigación por parte de uno de los docentes es mediante lecturas previas referentes al tema que se va a trabajar. No obstante, los otros docentes observados basan la enseñanza de las matemáticas, cada vez que explican un tópico, en la realización de muchos ejercicios, con lo que los estudiantes se convierten en actores que no aportan a la clase. Cabe resaltar que el docente que promueve la participación constante de los estudiantes tiene más aceptación en el aula, un factor que resulta fundamental para la creación de un ambiente agradable.

Si se tiene en cuenta lo propuesto por el MEN (2016), que afirma que un docente del siglo XXI debe ser proactivo y capaz de encontrar alternativas que le permitan tomar el rol de líder de la clase en búsqueda de brindarles a sus estudiantes las mejores condiciones para que sean más eficaces, se encuentra que la realidad no se corresponde con el constructo teórico, pues los egresados del programa no están usando la investigación para construir clases transformadoras.

Entrevista: en la entrevista aplicada a los docentes, estos coinciden al expresar que, a pesar de que la formación recibida en investigación durante su época de estudiantes no fue la mejor, sí están convencidos de que esta es de vital importancia en el desarrollo de su práctica pedagógica diaria. A su vez, son claros en afirmar que deben comprometerse más en involucrar la investigación a sus clases. Sobre todo teniendo en cuenta que, con base en Tejada-Fernández, Carvalho-Dias y Ruiz-Bueno (2017), la investigación genera impacto en la práctica de los docentes, al tiempo que fortalece las competencias científicas en el caso de los docentes de matemáticas. No obstante, los docentes egresados no utilizan la investigación como medio para generar impacto en la educación. Ahora bien, las limitantes que mencio- 
nan los docentes con respecto a su formación investigativa no es impedimento para uno de ellos, quien mencionó que, en algunas ocasiones, ha fomentado la investigación en el aula, particularmente desde la lectura, y que uno de sus mayores deseos es tener más conocimientos investigativos para ponerlos en práctica con sus estudiantes.

Triangulación de la información de bitácora de observación, diario de campo y entrevistas: al triangular la información, resulta claro que para los docentes es de vital importancia tener nociones investigativas y aplicarlas en su práctica constante. Aseguran que es una de las competencias con las que deben contar, y también son sinceros al manifestar que esta práctica no es utilizada por la mayoría de ellos; aducen como principal causa de esto la poca formación que recibieron en su etapa de estudiantes del programa de licenciatura en Matemáticas, en donde solo sintieron la necesidad de investigar cuando realizaron el trabajo de grado, es decir, casi al final de sus estudios. Pese a esto, según lo observado, un docente de los entrevistados asume el rol de ser docente-investigador con base en las actividades que planifica en sus clases.

\section{Categoria: práctica pedagógica}

Bitácora de observación: a partir de las observaciones realizadas, se pudo apreciar, respecto a la práctica pedagógica que tienen los docentes egresados, que estos no cuentan con repertorios de estrategias que les permitan ganar la atención de sus estudiantes en lo referente al conocimiento matemático. Sin embargo, es destacable que uno de los docentes interactúa más con sus estudiantes y utiliza técnicas como la lectura y la participación para capturar su atención en la clase, logrando elementos como la concentración y la motivación en el aprendizaje de la matemática; cosa que no ocurre con los otros docentes, quienes carecen de elementos que generen empatía en sus estudiantes, y estos, por tanto, no perciben las matemáticas de buena forma. 
Diario de campo: a través del diario de campo se pudo apreciar que los docentes egresados del programa no cuentan con herramientas de tipo didáctico que promuevan el crecimiento de su práctica pedagógica. Es claro que uno de los principales estímulos que puede recibir un estudiante es cuando su docente lo motiva a diario con cosas que, aunque parezcan mínimas, para él son relevantes. En ese orden de ideas, uno de los docentes se destaca por fomentar todo lo anterior y, de una u otra forma, consigue resultados desde lo atencional y actitudinal; no así los docentes que asumen roles tradicionales que no les permiten consolidar su práctica y cuyos estudiantes perciben sus clases como monótonas y carentes de importancia para su formación académica y como seres integrales.

Entrevista: en la entrevista realizada a los docentes egresados, estos son concretos al afirmar que necesitan fortalecer su práctica pedagógica. Reconocen también que no haber tenido una buena formación en la universidad con respecto al manejo de estrategias didácticas no es excusa y que, en la educación, se requiere renovación constante para lograr ser un docente exitoso. En esta línea, uno de los docentes pone de manifiesto que la práctica pedagógica se ve potenciada a través de la investigación, y agrega que si se explora, lee y crece en la academia, el repertorio de estrategias será aún más grande y, por tal motivo, la práctica pedagógica en la enseñanza de las matemáticas se verá beneficiada a través de las experiencias del aula.

Triangulación de la información de bitácora de observación, diario de campo y entrevistas: al triangular los tres instrumentos, cabe resaltar que hay concordancia con respecto a la información recolectada a través de la observación y el seguimiento a algunas clases de los docentes con respecto al discurso brindado en la entrevista. Esto, dado que la práctica pedagógica que llevan a cabo rutinariamente se basa en clases de tipo tradicional, ya que suelen usar escasos recursos de tipo didáctico. Con todo, son conscientes de que es algo que deben mejorar en búsqueda de su crecimiento como docentes. Cabe resaltar, no obstante, que uno de los docentes se destaca un poco más por innovar y ser menos 
tradicionalista, con el objetivo de que sus estudiantes se sientan más a gusto y perciban las matemáticas como algo divertido y no complicado para sus vidas.

\section{Categoria: relación entre investigación en educación matemática y práctica pedagógica}

Bitácora de observación: a partir de lo observado, se pudo concluir que la relación entre práctica pedagógica e investigativa que muestran los docentes egresados es casi nula, a pesar de que, a lo largo de los años, la investigación se ha tomado como una herramienta para fortalecer la práctica pedagógica de los docentes a partir de las experiencias del aula (Godino, 2011). Sin embargo, esto no se percibe desde el quehacer de los egresados del programa, pues en sus clases no se toma la investigación como un elemento importante de su práctica diaria. No obstante, existen esfuerzos para que ese panorama cambie, en especial, con un docente esforzado que implementa estrategias áulicas que le permiten tener una buena conexión con sus estudiantes.

Diario de campo: el seguimiento constante a los docentes egresados permitió percibir que los docentes egresados del programa de licenciatura en Matemáticas presentan falencias a la hora de darles una mirada a sus clases utilizando la investigación como elemento clave. Dado que el repertorio de estrategias que utilizan los docentes no suele ser llamativo para los estudiantes, se imposibilita, de una u otra manera, desarrollar investigación en el aula, pues los estudiantes son poco partícipes en las clases. Así mismo, a pesar de que existe un docente que hace intentos de relacionar su práctica con la investigación por medio de la exploración, los recursos o aprendizajes limitados que obtuvo en su pregrado evitan que pueda obtener resultados óptimos.

Entrevista: es importante decir que, durante la entrevista realizada, los docentes, en su totalidad, fueron claros al expresar que 
en la educación actual la práctica pedagógica va de la mano de la práctica investigativa. Como reflexión, agregaron que en su época de pregrado manejaban el mismo discurso, pues era lo que les solían decir, pero que no aplicar lo teórico de una u otra forma ha perjudicado su práctica, en el sentido de que sus clases se perciben como clases tradicionales en las que prima el saber matemático desde su esencia y no el fomento a aplicarlo en el contexto a través de exploraciones e investigaciones que pudieran ser provechosas teniendo en cuenta la época en la que nos encontramos. Así mismo, concluyeron que esperan seguir cualificándose en investigación en búsqueda de crecer en su práctica pedagógica.

Triangulación de la bitácora de observación, diario de campo y entrevistas: en cuanto a la relación entre investigación en educación matemática y práctica pedagógica, al triangular los tres instrumentos es importante afirmar que existe coherencia en el discurso de los docentes. Para ellos, es de suma importancia relacionar la práctica pedagógica con la parte investigativa en el aula, ya que esto permite que haya más argumentos para que el rendimiento y la motivación de sus estudiantes mejore. Sin embargo, son conscientes de que en sus clases no logran incluir esta relación, $\mathrm{y}$, por tal motivo, estas se vuelven tradicionales y de poco agrado para los estudiantes. Esto se evidencia en las observaciones y en el diario de campo que hicieron durante la recolección.

\section{Conclusiones}

1) Durante la investigación se alcanzó el objetivo planteado inicialmente de realizar una caracterización del rol docente en la investigación y su relación con la práctica pedagógica. Esto, desde la elaboración de un constructo que sirve de horizonte para alcanzar el ideal teórico propuesto en las directrices del Ministerio de Educación Nacional, para definir las competencias investigativas que son necesarias en aras de ser investigador en el contexto nacional. 
2) A través del análisis de la información recolectada entre los docentes egresados del programa que ejercen su profesión en una institución educativa, se pudieron confrontar sus visiones particulares y generales de los procesos investigativos y su relación con las prácticas pedagógicas. El perfil que tienen estos docentes no es precisamente el de investigadores, ni mucho menos el de docentes que promuevan investigar. Además, los docentes egresados del programa suelen no incluir la investigación en sus prácticas pedagógicas, debido a que la visión con la que se quedaron de sus docentes de pregrado no fue la mejor. Creen, por tanto, que ahí reside la gran causa de que su práctica pedagógica sea muy estática y de que el ambiente escolar en muchos casos no sea el mejor.

\section{Recomendaciones}

1) Se recomienda hacer revisiones y ajustes al perfil del docente que guiará las asignaturas de Investigación formativa, Investigación en educación matemática y Práctica pedagógica, teniendo en cuenta las consideraciones expuestas en este trabajo, así como lo establecido por el Ministerio de Educación Nacional y por Colciencias, que es el ente máximo para establecer el perfil de investigador a nivel nacional.

2) Además, se recomienda ajustar las cartas descriptivas para que sean coherentes con lo recomendado por el Ministerio de Educación Nacional en el decreto 2450 del 17 de diciembre del 2015, la Resolución 9317 del 6 de mayo del 2016, la Resolución 02041 del 2016 y los Lineamientos de calidad para las licenciaturas en educación publicados el 5 de mayo del 2014.

3) Se recomienda el trabajo grupal entre los docentes del programa de licenciatura en Matemáticas y de las asignaturas de Investigación y Práctica pedagógica, con el fin de conformar un espacio en el que se debata y se compartan experiencias e ideas 
que estén relacionadas con estas asignaturas, y de crear, así, un proceso a largo plazo con los maestros en formación inicial durante cada semestre cursado.

4) Fomentar la investigación desde el aula de clase, como un proceso inherente a la práctica pedagógica en todos los niveles de formación. Esto, a través del impulso que le brinden los docentes con publicaciones de artículos y con una hoja de vida que evidencie que se está trabajando como un docente investigador.

\section{Referencias}

Acuña, A. (2015). ¿Formar en investigación? ¿Enseñar a investigar? Una reflexión para el debate. En Memorias Simposio Internacional de Educación, Pedagogía y Formación. Innovaciones y educación para la paz (pp. 155-162). Cartagena: Universidad de Cartagena - Redipe.

Arboleda, L. C. y Castrillón, G. (2007). Educación matemática, pedagogía y didáctica. REVEMAT- Revista Eletrônica de Educação Matemática, 2(1), 5-27.

Badillo, E., Azcárate, C. y Font, V. (2011). Análisis de los niveles de comprensión de los objetos $\mathrm{f}^{\prime}(\mathrm{a})$ y $\mathrm{f}^{\prime}(\mathrm{x})$ en profesores de matemáticas. Enseñanza de las ciencias: revista de investigación y experiencias didácticas, 29(2), 191-206.

Badillo, E., Font, V. y Martínez, M. (2013). Visualización gráfica y análisis comparativo de la práctica matemática en el aula. Enseñanza de las ciencias. Revista de investigación y experiencias didácticas, 31(3), 207-225.

Edo, M. y Badillo, E. (2014). Enseñar y aprender matemáticas en los prácticums del Grado de Educación Infantil de la UAB. UNO: Revista de Didáctica de las Matemáticas, (66), 36-46.

Fernández, C., Llinares, S. y Valls, J. (2012). Learning to notice students' mathematical thinking through on-line discussions. ZDM Mathematics Education, 44(6), 747-759. 
Garcés, R. (2010). El rol del docente en el contexto actual. Revista Electrónica de Desarrollo de Competencias (REDEC), 2(6).

Giménez, J., Vanegas, Y., Font, V. y Rubio, N. (2016). Ciudadania y Formación de Profesores de Matemáticas. Recuperado de ResearchGate https://www. researchgate.net/publication/305619733

Godino, J. D. (2011). Inicio a la investigación en la enseñanza de las matemáticas. En J. M. Goñi, J. D. Godino, N. Planas y V. Font (Eds.), Matemáticas, investigación, innovación y buenas prácticas (pp. 9-55). España: Secretaría General Técnica, Centro de Publicaciones, Ministerio de Educación - Editorial GRAÓ, de IRIF, S.L.

Hernández, I. (2009). El docente investigador en la formación de profesionales. Revista virtual Universidad Católica del Norte, (27), 1-21.

Hernández, R. (2003). Metodología de la Investigación. México: McGraw-Hill Interamericana Editores.

Hernández, R. (2010). Metodología de la Investigación. México: McGraw-Hill Interamericana Editores.

Llinares, S. (2014). Enseñar matemáticas y aprender a mirar de forma profesional la Enseñanza. Conocimiento y emociones del profesorado. En A. Perafán, E. Badillo y A. Adúriz-Bravo, Conocimiento y emociones del profesorado. Bogotá: Paidós.

Mallart Solaz, A., Font Moll, V. y Malaspina, U. (2016). Reflexión sobre el significado de qué es un buen problema en la formación inicial de maestros. Perfiles Educativos, XXXVIII(152), 14-30.

Ministerio de Educación Nacional. (2005). Ser maestro hoy. El sentido de educar y el oficio docente. Al Tablero, (34).

Ministerio de Educación Nacional. (2014). Lineamientos de calidad para las licenciaturas en educación. Bogotá: Ministerio de Educación Nacional. 
Ministerio de Educación Nacional. (2015). Decreto 2450. Bogotá.

Ministerio de Educación Nacional. (2016). Resolución N. 02041. Bogotá.

Pochulu, M. y Rodríguez, M. (Comps.). (2012). Educación Matemática - Aportes a la formación docente desde distintos enfoques teóricos. Los Polvorines: UNGS y EDUVIM.

Pochulu, M., Font, V. y Rodriguez, M. (2016). Desarrollo de la competencia en análisis didáctico de formadores de futuros profesores de matemática a través del diseño de tareas. Revista latinoamericana de investigación en matemática educativa, 19(1), 71-98.

Ramírez, M. S. (2008). Triangulación e instrumentos para análisis de datos [video]. Recuperado de https://www.youtube.com/watch?v=0OG_OLBT_VA

Restrepo, B. (2005). Conceptos y aplicaciones de la investigación formativa, y criterios para evaluar la investigación científica en sentido estricto. Recuperado de http://www.epn.edu.ec/wp-content/uploads/2017/03/ Investigaci\%C3\%B3n-Formativa-Colombia.pdf

Riscanevo Espitia, L. E. y Jiménez Espinosa, A. (2017). La experiencia y el aprendizaje del profesor de matemáticas desde la perspectiva de la práctica social. Praxis y saber. Revista de Investigación y Pedagogía, 8(18), 193-222.

Saker García, J. (2014). Práctica pedagógica investigativa en las Escuelas Normales Superiores: contexto y pertinencia de la calidad educativa. Educación y Humanismo, 16(26), 83-103.

Sánchez-Robayo, B. y Torres-Duarte, J. (2017). Aprender a investigar investigando. Realización de una propuesta de formación. Revista científica, 1(28), 17-32.

Schoenfeld, A. y Kilpatrick, J. (2008). Toward a theory of proficiency in teaching mathematics. En Tools and processes in mathematics teacher education (pp. 321-354). Rotterdam, Netherlands: Sense publishers. 
Tejada-Fernández, J., Carvalho-Dias, M. y Ruiz-Bueno, C. (2017). El prácticum en la formación de maestros: percepciones de los protagonistas. Magis. Revista Internacional de Investigación en Educación, 9(19), 91-114.

Vaillant, D. (2016). El fortalecimiento del desarrollo profesional docente: una mirada desde Latinoamérica. Journal of Supranational Policies of Education, (5), 5-21.

Valbuena Duarte, S., Conde Carmona, R. J. y Ortíz Ortíz, J. D. (2018a). La Investigación en educación matemática y Práctica Pedagógica, perspectiva de licenciados en Matemáticas en formación. Educación y Humanismo, 20(34), 201-215.

Valbuena, S., Conde, R. y Ortíz, J. (2018b). Perfil de formadores que administran módulos de Investigación y práctica en ciencias sociales y humanas. Revista Logos Ciencia y Tecnología, 10(2), 56-65.

\section{Cómo citar este artículo}

Valbuena Duarte, S., Conde Carmona, R. J. y Padilla Escorcia, I. A. (2018). La práctica pedagógica en la investigación en educación matemática desde la perspectiva de los egresados. Universitas Humanística, 86, 249-273. https:// doi.org/10.11144/Javeriana.uh86.ppie 